\title{
Karyokinetic Spindles and the Behavior of Centrioles in the Spermatocyte Meiosis of Silkworms and Grasshoppers
}

\author{
Yoshio Nakao, Yuh H. Nakanishi \\ National Institute of Radiological Sciences, Chiba, Japan \\ and \\ Bungo Wada ${ }^{1}$
}

Received April 5, 1967

The disappearance of nuclear membrane in late prophase and the disappearance of achromatic figures shortly after the completion of division and their de novo appearance in the succeeding division cycles are generally accepted as facts in current cytology text-books. However, it is surprising that the above generalization still exists. This enigma on mitotic figures in classical cytology has been discussed and revised in a monograph "Analysis of Mitosis" by Wada (1966), in which the experimental data presented were obtained primarily from in vivo observations on plant cell mitoses. Therefore, few descriptions on centrioles appeared in this monograph.

Recently, Went (1966) has written the monograph "The Behavior of Centrioles and the Structure and Formation of the Achromatic Figure". His treatise cites exhaustively data on the behavior of centrioles and the structure and formation of the mitotic apparatus in various kinds of organisms. Old descriptions are reviewed as well as more recent ones based on modern techniques such as physical methods, chemical methods, immunochemical treatments or electron microscopy. However, the writer's ideas on the developmental process of the mitotic apparatus seem to differ little from the classical cytology of the 1880's. Little consideration has been given to the importance of intracellular membranous structures existing as spindle membrane in the mitotic cell and of the cause-and-effect relations among continuous morphological changes of mitotic organelles demonstrated from in vivo observations. In spite of many evidences given by modern techniques cited in the monograph, the raison d'etre of centrioles remains still unsolved biologically.

In this paper the behavior of centrioles has been reinvestigated by studying the spermatocyte meiosis of silkworms and grasshoppers. A new interpretation on the role of centrioles is proposed from a comparison of the formation of sperm-tail filaments in these insects. In addition, in order to generalize on the mechanism of mitosis in plants and animals we have 1 Present address: 15-7, Kamiyama-cho, Shibuya-ku, Tokyo, Japan. 
reinterpreted some of the divergent achromatic figures described in classical cytology.

\section{Materials and methods}

The 2 species used in this study were the "Abrako" race of the silkworm, Bombyx mori, and the grasshopper, Chloealitis geniculaributs. Silkworm testes were taken from the larva on the second day of the fifth instar. The material was immersed for 15 minutes in $1 / 10 \mathrm{M}$ metal salt solution as pretreatment. The following metal salt solutions were tested: $\mathrm{CdCl}_{2}, \mathrm{CoCl}_{2}, \mathrm{~Pb}\left(\mathrm{NO}_{3}\right)_{2}, \mathrm{SnCl}_{4}$ and $\mathrm{MnSO}_{4}$. For post-fixation the material was transferred to Allen's PFA-3 fluid (picric acid, formalin and acetic acid $75: 15: 10$ by volume and $1 \mathrm{~g}$ of urea). The fixed testes were sectioned and stained with Heidenhain's iron alum haematoxylin according to the routine paraffin method. In vivo observations were studied comparing living cells with fixed ones in order to grasp correctly the entity of centrioles and the appearance of achromatic figures.

For the grasshoppers, the testes were fixed with Bouin's fluid after pretreatments with the same metal salt solutions mentioned above. The fixed material was sectioned $10 \mu$ and $21 \mu$ thick and stained with Heidenhain's iron alum haematoxyline.

\section{Results}

\section{Silkworm spermatocyte meiosis}

The presence of flagella was demonstrated by time lapse photography, in the living spermatocyte meiosis of silkworm (Figs. 1-4). The cell shown in Figs. 1-4 were photographed at different times. At the upper region of the cell (Figs. 1 and 2) one long flagellum, probably one of a pair of flagella, is visible and at the lower pole of the spindle in Fig. 1, a pair of flagella are visibile. At the basis of each flagellum a granule, probably composed of two centrioles, can be observed. This granule lies apart from the spindle pole, although the direct connection of the flagellum with the granule is often concealed by a thin halo, a bright circle of Beck line, which inevitably appears surrounding the cell body in the fluid medium under the phase contrast microscope.

By using the metal salt solution fixatives, the atractoplasm of silkworm metaphase spindle appears to be independent of the cytoplasm and the centrioles lying at the periphery of the cell behave as basic bodies of each flagellum (Figs. 5 and 6). Even when the centrioles seem to stick to the spindle pole, they are separated by the spindle membrane and lie on its outer surface. The cell A in Fig. 5 shows a metaphase spindle whose spindle axis is slightly declined, so that the spindle membrane appears as a part of a circular line at the optical section of the spindle cone. The cell $\mathrm{B}$ in the same photograph shows a flagellum elongated from the centriole and the 

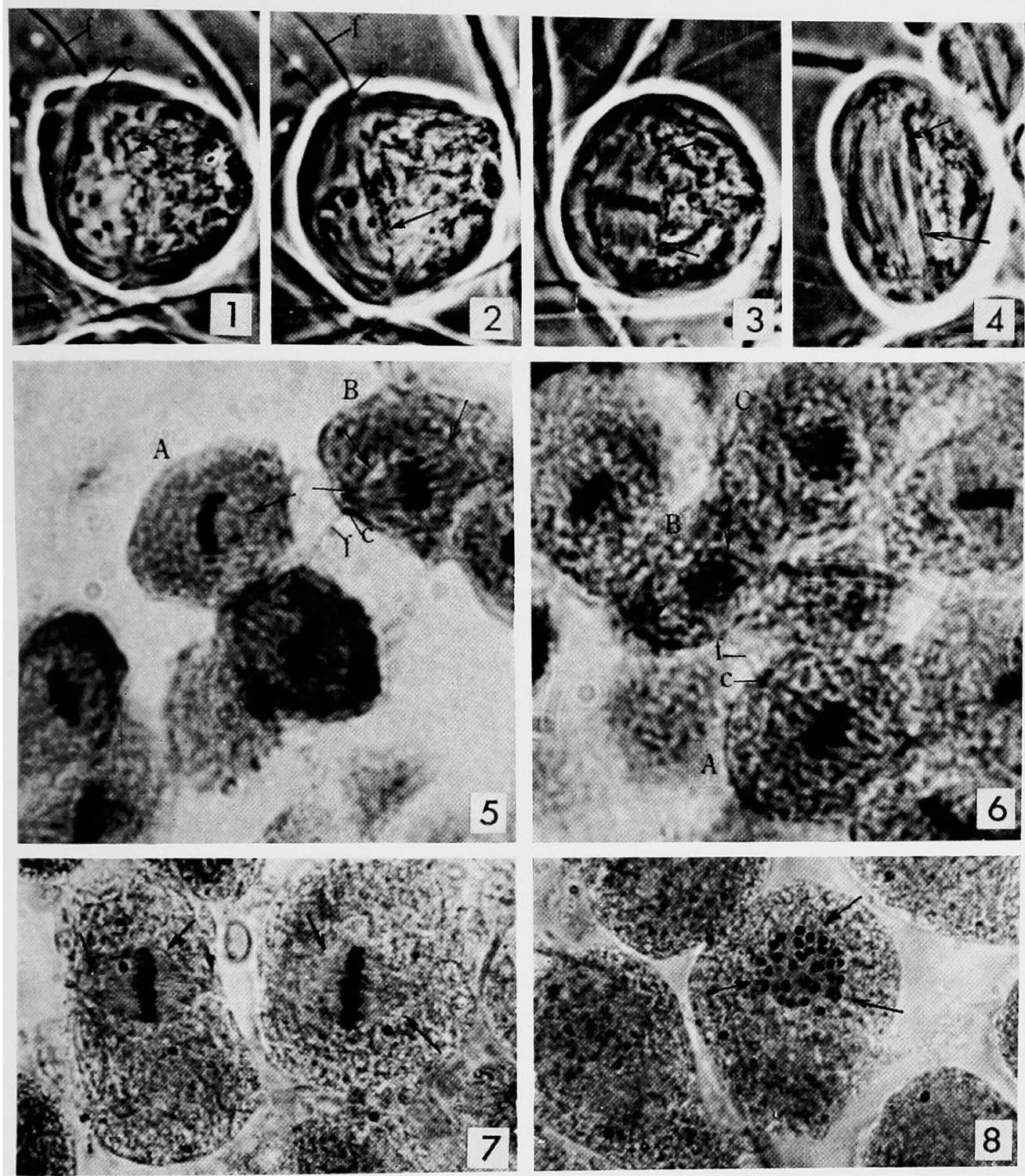

Figs. 1-8. Meiotic figures of spermatocytes in silkworms, Bombyx mori. Arrows indicate positions of nuclear membrane or spindle membrane. 1-4, time lapse photographs of a spermatocyte cell mounted in a pool of body fluid derived from the operated animal. Photographed with phase contrast microscope. Numerals in parentheses give times photographed. Temperature $25^{\circ} \mathrm{C}$. 1, late prophase $(6: 05)$. 2, prometaphase (6:09). 3, metaphase $(7: 21)$. 4, telophase $(8: 29)$. 5-6, spermatocyte meiosis fixed with Allen's fluid PFA-3 after pretreatment with $\mathrm{CoCl}_{2}$ solution. 5, spindle membrane of cell A whose spindle axis slightly declines, appears as a part of circular line. Centrioles and a flagellum of cell B lie at cell periphery apart from spindle poles. 6, in cell A in early anaphase, cell $B$ in prometaphase and cell $C$ in late prophase, nuclear membranes are visible and space occupied by the atractoplasm are distinguishable from cytoplasm. 7-8, spermatocyte meioses treated with acetocarmine squash method. Spindle membrane are traceable, when attention is paid to intracellular membranous structures. 7 , side view of metaphase spindles. 8, polar view of metaphase spindle. $\mathrm{f}$ : flagellum, c: centriole. 
independence of the spindle body from the cytoplasm. It also shows a part of the spindle membrane. At the cell $\mathrm{A}$ in Fig. 6, a flagellum develops from one of the centrioles lying in the periphery of one cell.

In spite of the fact that the spindle membrane is sometimes preserved in ordinary acetocarmine squash preparations, the presence of the spindle membrane and its biophysical importance have been disregarded in classical cytology and even in current cytology text-books. For instance, the cells in Figs. 7 and 8 (acetocarmine squash) show the presence of spindle membrane and the independence of the atractoplasm from the cytoplasm. The arrows in the photographs indecate the positions of the spindle membrane. Side views of the metaphase spindles are shown in Fig. 7 ; a polar view is shown in Fig. 8.

In short, the presence of spindle membrane has been disregarded in classical cytology, because not only of its fragile structure against routine fixatives but also because cytomorphologists failed to realize the functional importance of intracellular membranous structures. The data presented above clearly demonstrate that the centrioles in the silkworm spermtocyte meiosis are cytoplasmic in origin and primordial organelles for flagellum formation and they have no relations to the spindle formation.

2. Grasshopper spermatocyte meiosis

In the case of grasshopper spermatocyte meiosis, the role of the centrioles as primordial organelle for flagellum formation first appears in the spermatid stage in which the sperm-tail filament grows forth from the centriole by transformation and by association of nebenkern, chondriosomes and other cytoplasmic inclusions.

Figs. 9-11 show the behavior of centrioles in a grasshopper spermatocyte meiosis in metaphase I treated with metal salt solution fixatives. Figs. 9, 10 and 11 are photographs of the same karyokinetic metaphase spindle taken at three different focal levels of the microscope. In Fig. 9, the focus is set on the spindle membrane at the upper pole of the spindle. Here the centrioles lie apart from the pole in the cytoplasm. Astral rays grwon from the centrioles cover the spindle and make slightly indistinct the spindle membrane at the pole. But there is no reason to disregard the presence of the spindle membrane. When the focus is on the centrioles (Fig. 10), the spindle membrane obscure, although the space occupied by the atractoplasm is clearly distinguishable from the cytoplasm. In in vivo observations on the grasshopper spermatocyte meiosis, Podisma sapporense, Makino and Nakanishi (1955) have already pointed out that the spindle is clearly distinguishable from the surrounding cytoplasm. In Fig. 11, the focus is on the astral rays. These rays thickly cover the upper polar region of the spindle and conceal the spindle membrane. The chromosomal fibers of a chromosome lying at the middle of the spindle body orient to the both spindle poles. In the lower pole, the fiber arrives and ends at the indise of the spindle membrane. 
Fig. 12 shows a side view of the spindle membrane in metaphase II. In this cell, a pair of centrioles are visible at the upper pole outside the spindle membrane. A schematic drawing of the fine structures of the metaphase spindle from Fig. 12 is shown in comparison of the so-called mitotic apparatus in classical cytology (Fig. 15A and 15A'). In Fig. 14, it can also be seen that the centrioles lie outside the spindle membrane. In most cases, however, centrioles stick, probably by adsorption, to the outer surface of the spindle membrane and move poleward along to it and stop at the spindle pole. Figs. 13 and 14 are photographs of the same cell at metaphase II taken at different foci. The arrows in the photographs indicate the positions of the spindle membrane. The spindle membrane in Fig. 13 appears as an elliptic circular line at the optical section of the body, because the spindle axis declines to the optical section of the microscope. The upper pole of the spindle lies out of focus. Fig. 14 shows the centrioles at the upper pole of the spindle, toward which the chromosomal fibers converge. A few astral rays radiate around the centrioles to the cytoplasm. These features make it difficult to distinguish the astral rays from the spindle fibers and to recognize the outline of the spindle body.

From a comparison of the centriole behavior in the silkworm spermatocyte meiosis with the centriole behavior in grasshoppers, it becomes clear that the intrinsic role of centrioles is concerned with the sperm-tail filament formation which occurs in the silkworms at the spermatocyte stage and in the grasshoppers at the spermatid stage. However for both kinds of insects no centrioles participate in the spindle formation.

\section{Divergent achromatic figures}

To generalize on a common mechanism of mitosis for plants and animals, we have reconsidered some divergent achromatic figures described in classical cytology from the standpoint of the atractoplasm theory and imagined their fine structures based on the new blueprint for mitosis (Wada 1966).

In current cytology text-books, the independent spindle unit chromosomes in the first meiotic metaphase in the cocoid, Llaveia bourari (HughesSchrader 1931), the non-convergent anaphase spindle in the first meiotic division in maize (Clark 1940), and the monopolar spindle in the first spermatocyte division of the diptera, Sciara coprophila (Metz 1933) are often cited as examples of divergent achromatic fibures. These divergent achromatic figures are difficult to explain based ordinary spindle mechanisms, where neither centrioles nor spindle poles appear during karyokinesis. The presence of a spindle membrane has usually been disregarded by cytologists.

Studies on the use of kinds and amounts of metal salt solutions for the fixation of the spindle membranes will result in the demonstration of the presence of spindle membrane even in the divergent achromatic figures shown in Figs 15 $\mathrm{B}^{\prime}, 15 \mathrm{C}^{\prime}$ and 16 . The presence of the spindle membrane, namely an intracellular membranous structure, is an indispensable condition for the 

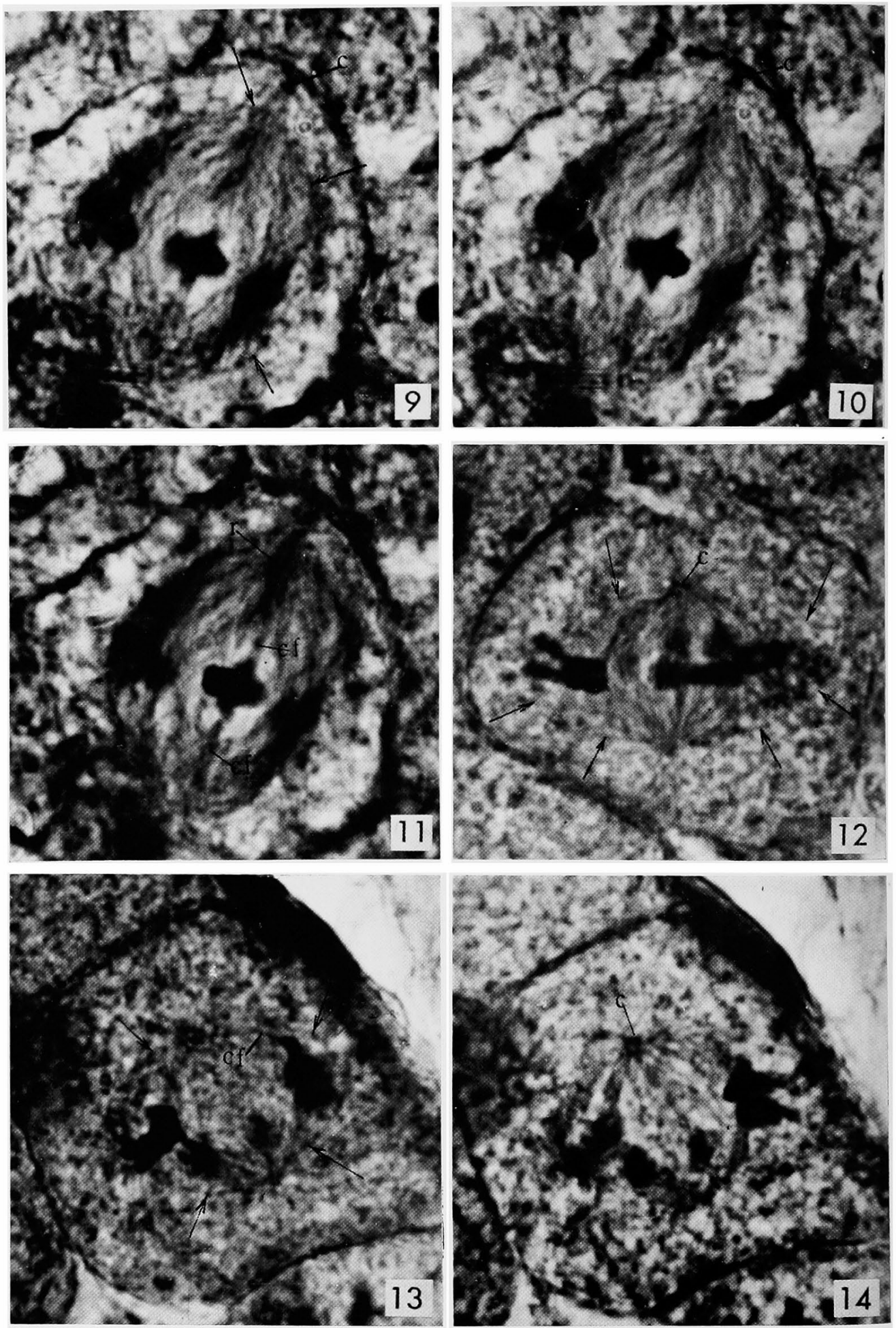

Figs. 9-14. Meiotic figures of spermatocytes in grasshopper, Chloealitis geniculaributs, fixed with Bouin's fluid after pretreatment with $\mathrm{MnSO}_{4}$ solution. Arrows indicate positions of spindle membranes. 9-11, a metaphase cell photographed at three different focal levels. 9 , showing spindle membrane at upper pole of spindle. 10 , centriole lying at cell 
poleward movement of chromosomes or the shortening of the chromosomal fibers: all the energy transfers for the chromosome movement are physi-
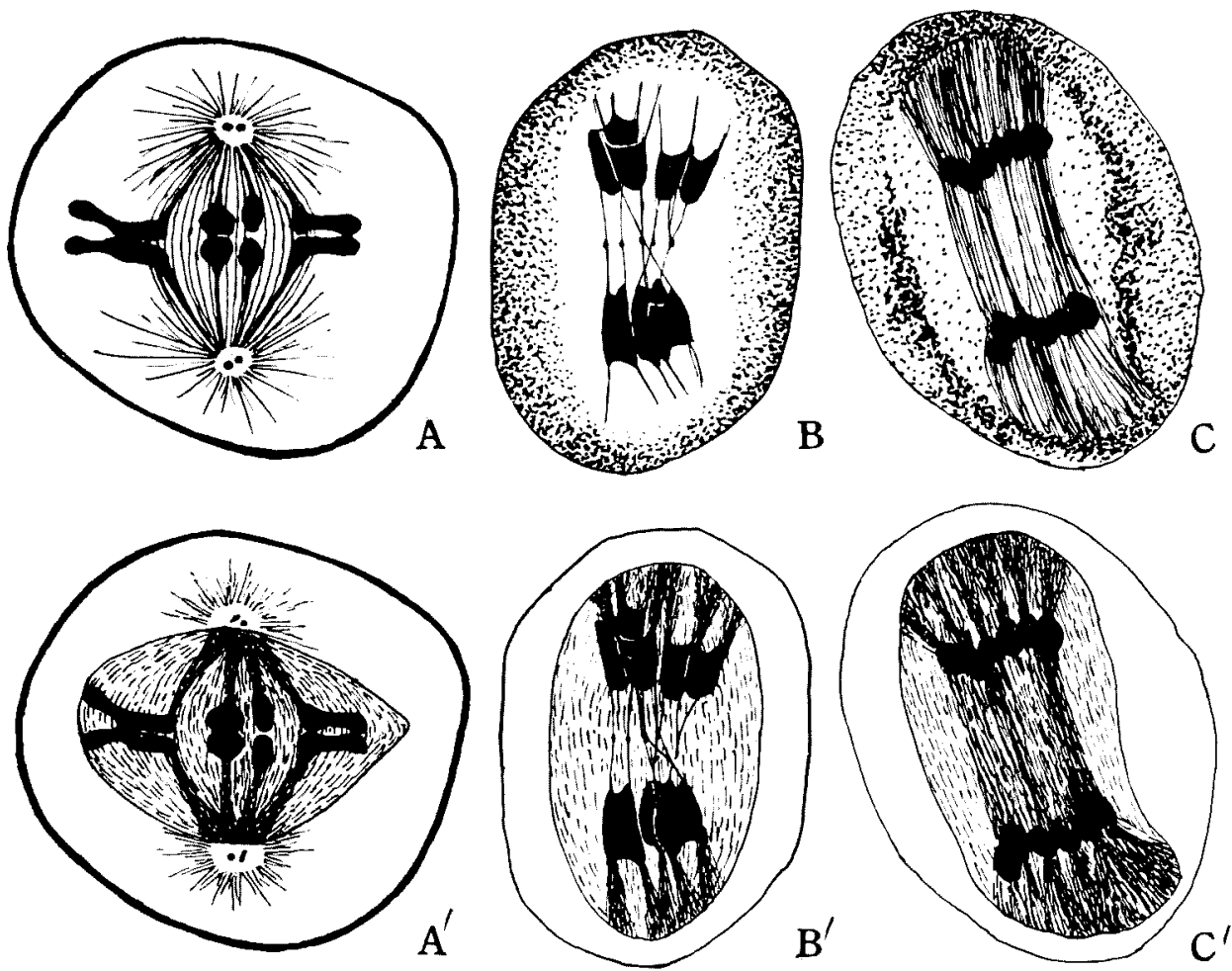

Fig. 15. Schematic diagram showing normal and divergent achromatic figures. $A$ and $A^{\prime}$, show second meitotic metaphase in grasshopper spermatocyte, Chloealitis geniculaributs, drawn after photograph in Fig. 12. A, represents normal metaphase spindle drawn according to common ideas in classical cytology. $\mathrm{A}^{\prime}$, illustrating fine structures of atractoplasm, chromosomal fibers and presence of spindle membrane based on atractoplasm theory. Centrioles and astral rays lie outside the spindle membrane in cytoplasm. B and C, showing first meitotic division in Llaveia bouvari (Hughes-Schrader 1948) and that in maize, Zea mays, with a recessive gene $d v / d v$ (Clark 1940) drawn after original papers. $\mathrm{B}^{\prime}$ and $\mathrm{C}^{\prime}$, illustrating fine structures of divergent achromatic figures, chromosomal fibers and presence of spindle membrane based on atractoplasm theory. Comparing $\mathrm{B}$ with $\mathrm{B}^{\prime}$, it cannot be decided until after the confirmation of perfect fine structures of achromatic figures, whether each chromosome represents independent spindle unit or dicentromeric chromosome with two chromosomal fibers. Divergent spindle fibers in $\mathrm{C}$ are suggested to end inside the spindle membrane as drawn in $\mathrm{C}^{\prime}$, provided achromatic figures would be treated with improved fixatives and spindle membrane would be preserved in fixed preparations.

periphery shows no direct connection to spindle organization. 11, bundle of astral rays which cover the upper pole of spindle, conceal the upper terminals of chromosomal fibers, while the lower terminals is visible inside the spindle pole. 12, a side view of spindle in metaphase II. Two centrioles lie closely to the outside of upper pole of spindle. 1314 , a spindle body in metaphase II at different foci. 13, showing an optical section of declined spindle body. The spindle membrane at optical section appears as an elliptic circular line. 14, centrioles which lie out of focus in Fig. 13 are visible outside of spindle pole. c: centriole, cf: chromosomal fiber, $r$ : astral rays. 


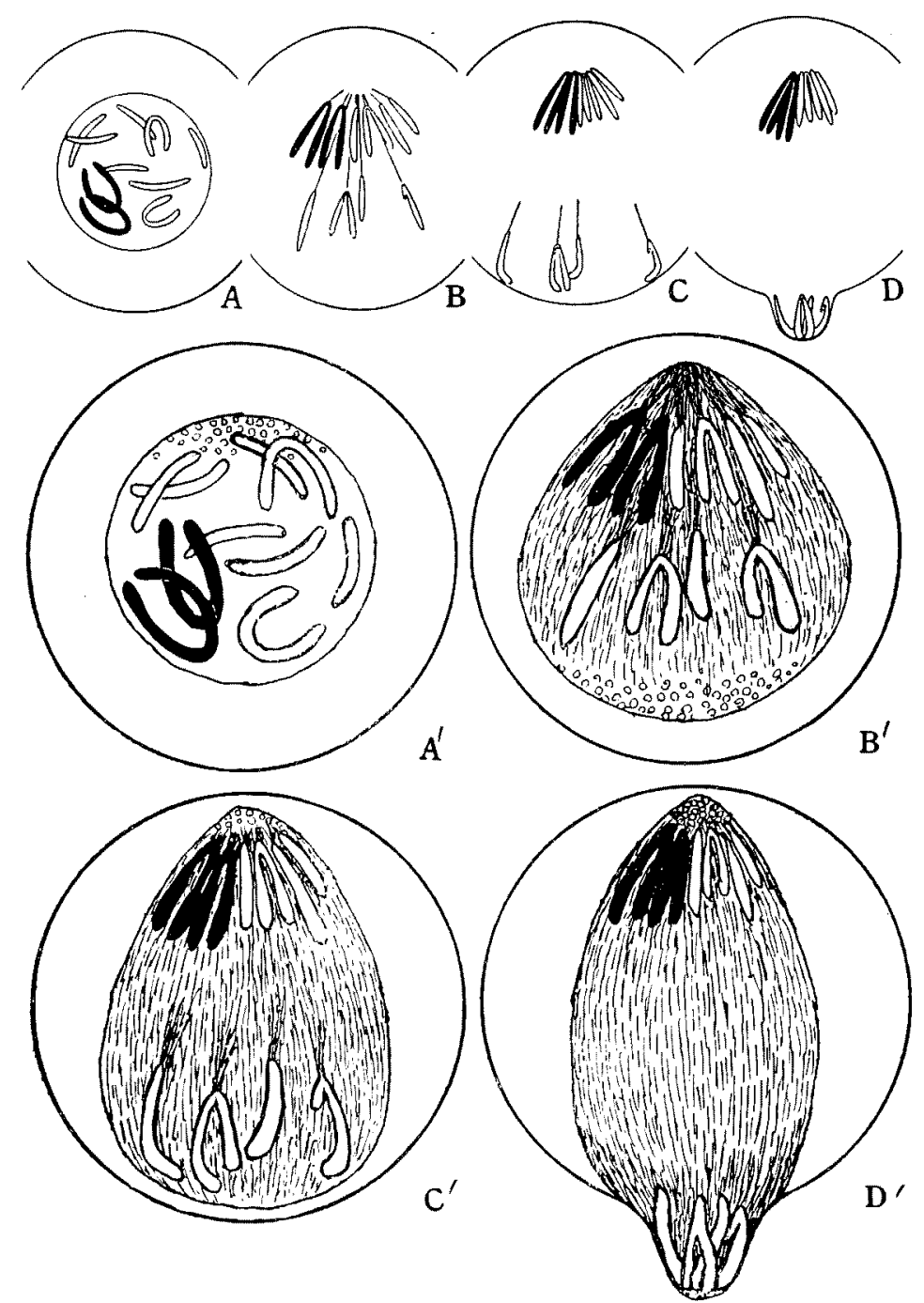

Fig. 16. Schematic diagram showing first spermatocyte division in Sciara coprophila. A, B, C, and D are drawn after original paper (Metz 1933). $\mathrm{A}^{\prime}, \mathrm{B}^{\prime}, \mathrm{C}^{\prime}$ and $\mathrm{D}^{\prime}$ illustrate the fine structure of each monopolar spindle based on atractoplasm theory. $\mathrm{A}^{\prime}$, late prophase showing initial appearance of fibril precursors only in one side of nuclear membrane where spindle monopole will develop. $\mathrm{B}^{\prime}$, early anaphase. All chromosomal fibers converge to one and the same spindle pole. Chromosomes of one homologous chromosome set lying near monopole migrate toward the pole, while chromosomes in the other set with short, immature chromosomal fibers are pushed by fibrillation of fibril precursors toward the opposite side of the monopole. $\mathrm{C}^{\prime}$, by shortening of chromosomal fibers, consequently by refolding of each fibril, chromosomes of one set reach the pole, while those of other set being pushed by continuous fibrillation, come in contact with the spindle mem. brane. $\mathrm{D}^{\prime}$, showing chromosome sets in telophase and beginning of abnormal cytokinesis at opposite side of monopole. Small rings connote fibril precoursors in Figs. $\mathrm{A}^{\prime}$ and $\mathrm{B}^{\prime}$ and refolded fibrils in Figs. $\mathrm{C}^{\prime}$ and $\mathrm{D}^{\prime}$. cochemically performed by the intervention of the spindle membrane. Practically, the spindle membrane is functionally an essential organelle for spindle activity, just as the plasma mem. brane, or cell membrane, is for cell activity, although both membranes are morphologically so delicate and fragile that their realization in fixed preparations has been left technically as difficult problems.

To the unique movement of chromosomes in the Sciara meiosis, as it seems to be difficult to understand the chromosome behavior only by the presence of spindle membrane, a few words will be added on the possible mechanism of the chromosome movement in a 
monopolar spindle (Fig. 16).

In the case of bipolar spindles, no matter whether the poles are pointed or flat, the chromosome arrangement of metaphase plates takes place inside the spindle membrane. The accumulation of fibril precursors at both spindle poles and their fibrillation proceed in one direction from both poles to the equator of the nucleus pushing all the chromosomes lying in the path toward the equator but never going beyond the equator. See details in the monograph (Wada 1966, pp. 92-96).

In the spermatocyte meiosis of Sciara, only one spindle pole appears, so that the accumulation of fibril precursors and their fibrillation proceed one-sidedly from the monopole to the opposite side of the nucleus (Figs. $16 \mathrm{~A}^{\prime}$ and $\left.16 \mathrm{~B}^{\prime}\right)$. During this internal change, the prophase nucleus develops into a monopole spindle and all the chromosomes lying on the path of fibrillation are pushed to the opposite side of the spindle monopole (Figs. $16 \mathrm{~B}^{\prime}$ and $\left.16 \mathrm{C}^{\prime}\right)$. On the other hand, each chromosomal fiber elongates regularly from the kinetochore to the spindle pole. There is no reason to assume any difference between the oridinary bipolar spindles and this monopolar spindle with regard to the dispersed phase of atractoplasm colloidal sol and the presence of chemotaxis-like reaction between the kinetochores of each chromosome and the spindle pole.

In the case of the monopolar spindle in the Sciara meiosis, one of homologous chromosomes happens to lie nearer to the spindle pole than to the other set (Fig. 16B). Then, one chromosome set may finish the formation of chromosomal fibers earlier than the other set. Accordingly, it is assumed that the chemotaxis-like reaction between the single pole and the kinetochores would be saturated by the completion of chromosomal fibers of one chromosome set lying near the pole. Consequently, the chromosomal fibers of the other set develop weakly and short fibers appear faintly without reaching the monopole (Figs. $16 \mathrm{~B}^{\prime}$ and $16^{\prime} \mathrm{C}$ ). Therefore, only one set of the homologous chromosomes is pulled to thepole by shortening of the chromosomal fibers. On the other hand, the chromosomes of the other set with immature fibers are pushed far toward the opposite side of the monopole by continuous fibrillation of fibril precursors. Finally the chromosomes are arrested by the spindle membrane (Fig. 16C') and pressed to the inside of the membrane (Fig. 16D').

All the events suggested above cannot be explained without the presence of the spindle membrane. We believe that the presence of the spindle membrane in Sciara meiosis will be demonstrable by looking for adequate fixatives effective to preserve the spindle membrane of this material.

As in the case of spermatocyte meiosis in silkworms and grasshoppers, we feel that the various divergent mitoses found in different kinds of organisms may be explained on the principle of the atractoplasm theory. For this purpose, the confirmation of the presence of spindle membrane for each 
material takes precedence over any other kind of mitotic figures, such as the behavior of chromosomes, divergent features of spindles, flat spindle pole or the absence of centrioles. We expect that fixatives we have used will demonstrate the presence of the spindle membrane for materials that were previously reported to lack spindle membranes.

\section{Discussion}

Comparing the spermatocyte meiosis of silkworms with that of grasshoppers, no intrinsic difference was found concerning the entity of the karyokinetic spindles. They are nuclear in origin, behave as an independent system from the cytoplasm, and are enveloped with the spindle membrane derived from the nuclear membrane as already mentioned (Wada 1966).

In spite of the coincidence of karyokinetic spindle organization between silkworms and grasshoppers, the centrioles in both insects behave apparently in different way with regard to the sperm-tail filament formation. The silkworm centriole migrates to the periphery of the spermatocytes and develop into thin flagellum. The behavior of centrioles, especially with regard to their divisions and to the distributions among gametic cells as well as somatic ones, has been studied in detail by Tanaka (1955). He schematically illustrated various types of centriole divisions among insects, reptiles and mammals; and pointed out that the centrioles in male gametic cells of these animals ultimately develop into sperm-tail filaments.

From the results of papers cited by Tanaka and those of our observations, there is no doubt that the role of centrioles in silkworm spermatocyte meiosis is to prepare flagellum for sperm cell locomotion. Based on this fact, it may be natural to consider that the centriole in silkworm spermatocyte meiosis is nothing but a primordial organelle for flagellum formation. On the other hand, there is no confirmation of evidence that the centrioles in silkworm spermatocytes participate in the formation of karyokinetic spindle.

On the contrary, no transformation of centrioles into falgella takes place during the grasshopper spermatocyte meiosis, but this transformation appears, at first, at the spermatid stage. Tahmisian et al. (1956) studied in detail the grasshopper spermatogenesis and showed that the sperm-tail filaments initiate from the centrioles in the spermatids by association of nebenkern, chondriosomes, mitochondria and other cytoplasmic inclusions. In our observation with improved fixatives we have shown that the centrioles in the grasshopper spermatocyte meiosis behave always outside the spindle membrane and have no relation to karyokinetic spindle formation.

From the results of these investigations, we have reached the conclusion that the centriole of both insects is nothing but a primordial organelle for flagellum formation for the locomotion of sperm cells, and it may not be 
important whether the flagellum formation appears precociously in the spermatocytes or ordinarily in the spermatids. This difference might have been aquired during the evolutionary development of the organisms. Furthermore, the presence of centrioles and their divisions in mitosis in other than male gametic cells originate by the same way as in spermatocytes or spermatids, although no flagellum formation takes place. This fact suggests that the centrioles in other than male gametic cells remain in the cytoplasm neither entering into flagellum formation nor inducing cell locomotion. In other words, the centriole in other than male gametic cells exists in an inactive state.

The raison d'etre of centrioles will be reported in another paper where the behavior of centrioles will be compared among the unicellular organisms, metazoa and metaphyta, and the biological meaning of the disappearance of centrioles in highger plant cells discussed. The ultimate purpose of our investigations consists in the reinvestigation on the problems not only on centrioles but also on radiation biology or on cancer research which are linked directly to mitosis and cell proliferation.

\section{Summary}

1. The behavior of centrioles in spermatocyte meiosis of silkworms and of grasshoppers was reinvestigated by in vivo observations and by the use of improved fixatives.

2. In both insects, the centrioles behave exclusively outside the spindle membrane. The centrioles participate in the sperm-tail filament formation at the spermatocyte stage in silkworms and at the spermatid stage in grasshoppers. However, no centrioles participate in spindle formation.

3. Taking these facts into consideration, it is concluded that the centriole is intrinsically a primordial organelle for flagellum formation of the sperms and remains in an inactive state in other than male gametic cells in animals.

4. Based on the atractoplasm theory, suggestions are made concerning the mechanism of mitosis for some divergent achromatic figures and on the generalization of mitosis mechanisms in plants and animals.

\section{References}

Clark, F. J. 1940. Cytogenetic studies of divergent meiotic spindle formation in Zea mays. Amer. J. Bot. 27: 547-559.

Hughes-Schrader, S. 1948. Cytology of Coccids (Coccoidea-Homoptera). Advances in Genetics 2: $127-203$.

Makino, S. and Nakanishi, Y. H. 1955. A quantitative study on anaphase movement of chromosomes in living grasshopper spermatocytes. Chromosoma 7: 439-450.

Metz, C. W. 1933. Monocentric mitosis with segregation of chromosomes in Sciara and its bearing on the mechanism of mitosis. Biol. Bull. 64: 333-347.

Tahmisian, T. N., Powers, E. L. and Devine, R. L. 1956. Light and electron microscope studies of morphological changes of mitochondria during spermatogenesis in the 
grasshopper. J. Biophys. Biochem. Cytology 2: Suppl. 325-329.

Tanaka, K. 1955. Central body in the male reproductive cells of the silkworm with special reference to a peculiarity of centriole division in meiosis. Cytologia 20: 307-314.

Wada, B. 1966. Analysis of Mitosis. Cytologia 30: Suppl. 1-158.

Went, H. A. 1966. The Behavior of Centrioles and the Structure and Formation of the Achromatic Figure. Protoplasmatologia 6 (Gl): 1-109. 